\title{
B. GABUTTI
}

\section{A. OSTANELLO-BORREANI \\ The labeling method on two-dimensional networks}

Revue française d'automatique, d'informatique et de recherche opérationnelle. Recherche opérationnelle, tome 8, n V2 (1974), p. 17-29.

<http://www.numdam.org/item?id=RO_1974_8_2_17_0>

(C) AFCET, 1974, tous droits réservés.

L'accès aux archives de la revue « Revue française d'automatique, d'informatique et de recherche opérationnelle. Recherche opérationnelle » implique l'accord avec les conditions générales d'utilisation (http://www.numdam.org/ legal.php). Toute utilisation commerciale ou impression systématique est constitutive d'une infraction pénale. Toute copie ou impression de ce fichier doit contenir la présente mention de copyright.

\section{Numdam}

Article numérisé dans le cadre du programme

Numérisation de documents anciens mathématiques

http://www.numdam.org/ 


\title{
THE LABELING METHOD ON TWO-DIMENSIONAL NETWORKS
}

\author{
par B. Gabutti $\left({ }^{1}\right)$ et A. Ostanello-BorReani $\left({ }^{2}\right)$
}

\begin{abstract}
The Ford-Fulkerson labeling method has been extended, for solving the max-flow problem on two-dimensional networks, i.e. on networks with directed arcs on a cartesian plane $\mathbf{R}^{2}$. Computationally, the algorithm has a good efficiency for networks with at least one node of degree $\leqslant 3$ or with some symmetries.
\end{abstract}

\section{INTRODUCTION AND DEFINITIONS}

1.1. A two-dimensional network $G_{\vec{b}}=\left(X, A ;\left(\vec{b}_{j}\right)\right)$ is defined by a set $X,|X|=n$, of nodes $x_{i}$, a set $A,|A|=m+1$, of arcs $a_{j}=\left(x_{i}, x_{k}\right)$, and a family of $m+1$ directions $\vec{b}_{j}=\left(\alpha_{j}, \beta_{j}\right)$, one for each arc, i.e. of vectors of $\mathbf{R}^{2}$ such that $\alpha_{j}^{2}+\beta_{j}^{2}=1 \quad \forall_{j}$, [2].

The node-arc incidence matrix of $G_{\vec{b}}$ is the $2 n,(m+1)$ real matrix

$$
M=\left\{\vec{a}_{i j}\right\},
$$

whose general entry is defined as

$$
\vec{a}_{i j}=\left\{\begin{aligned}
\vec{b}_{j}^{\prime} & \text { if } a_{j} \text { leaves } x_{i} \\
-\vec{b}_{j}^{\prime} & \text { if } a_{j} \text { enters } x_{i} \\
\left(\begin{array}{l}
0 \\
0
\end{array}\right) & \text { if } a_{j} \text { is not incident to } x_{i}
\end{aligned}\right.
$$

where $\vec{b}^{\prime}$ is the transposed of $\vec{b}$.

(1) Laboratorio di Analisi Numerica (L.A.N.), Pavia, Italy.

(2) Politecnico di Torino, Torino, Italy.

This research was supported by the Gruppo Nazionale per l'Analisi Funzionale e le sue Applicazioni of the CNR (Consiglio Nazionale delle Ricerche) of Italy.

Revue Française d'Automatique, Informatique et Recherche Opérationnelle n mai 1974, V-2. 
1.2. Suppose that each $\operatorname{arc}(x, y) \in A$ has a given capacity $c(x, y)>0$ and that two nodes of $X$, say $s$ and $d$, are respectively the source and the sink for a flow « entering " $G_{\vec{b}}$ with a given direction $\vec{b}_{l}$, of the return $\operatorname{arc}(d, s)=a_{l}$.

A flow $\vec{f}$ on $G_{\vec{b}}$ is any vector of

$$
\mathbf{R}^{m+1} \quad, \quad \vec{f}=\left(f_{1}, f_{2}, \ldots, f_{m}, f_{l}\right),
$$

satisfying the conservation equations (or of static equilibrium) at the nodes

$$
\overrightarrow{M f}=\overrightarrow{0}
$$

Any node $x$, where (2) holds, is equilibrated. $f_{l}$ is the value of the flow $\vec{f}$, which is feasible if and only if

$$
\begin{aligned}
-c(x, y) & \leqslant f(x, y) \leqslant c(x, y) \quad \forall(x, y) \in A-\left\{a_{l}\right\} \\
c_{l} & =+\infty
\end{aligned}
$$

An obvious feasible flow is $\vec{f}=\overrightarrow{0}$. The problem of finding the maximum value of $f_{l}$ is the analogue of the max flow problem for the topological graph $G=(X, A)$.

The simplex method can obviously be used, since

$$
\begin{aligned}
& \text { « } \max f_{l} \\
& \text { such that (2) and (3) hold » }
\end{aligned}
$$

is a linear program.

1.3. The motivation of this problem comes from the study of optimum condition for the static equilibrium of planar pinned trusses with concentrated loads (see for example [9]).

An optimization problem, on such kind of structures, is that of evaluating the maximum loads supported by the structure under equilibrium conditions, given that the internal stress of any single bar cannot be greater then a known value.

For different kinds of structures, with different kinds of constraints and loads, the problem is reducible to a linear program (see for example [3], [5]).

1.4. A graph-theoretical approach of the problem has been performed in [2] and [8], and some classical results, such as the flow-path decomposition and the max flow-min cut theorem, have been found using a suitable definition of elementary path on $G_{\vec{b}}$, or $b$-path.

In this paper we intend to present an extension of the Ford-Fulkerson labeling method, [6], using the notion of $b$-path (or simply path in this context). For the paper be self-containing, we shall report the definition given in [2]. 


\section{2. b-PATH}

2.1. A $b$-path of $G_{\vec{b}}$ from $s$ to $d$ is $a$ subset $P \subseteq A$, connecting $s$ to $d$ and satisfying the rules given below.

Denote by $X(P)$ the set of nodes incident to the arcs of $P$.

Start : the return arc enters $s ; a_{l} \in P$.

Rule ( $(a)$ : If one arc $a_{j} \in P$ enters the node $x$ and the set, $\omega(x)$, of arcs incident to $x$, contains either

1) one arc $a_{k} \| a_{j}, a_{k} \notin P$, or

2) two $\operatorname{arcs} a_{k_{1}}\left\|a_{k_{2}}\right\| a_{j}, a_{k_{1}}$ and $a_{k_{2}} \notin P$, then $x \in X(P)$. The path leaves $x$ through $a_{k}$ or both $a_{k_{1}}$ and $a_{k_{2}}$.

Rule (b) : If two or more arcs not pairwise parallel of $P$ enter $x$ and $\omega(x)$ contains either

1) one $\operatorname{arc} a_{k} \notin P$, parallel to none of them, or

2) two arcs $a_{k_{1}} \frac{\| 1}{\|} a_{k_{2}} \| a_{j}, \forall a_{j} \rightarrow a_{j} \in P \cap \omega(x), a_{k_{1}}$ and $a_{k_{2}} \notin P$, then $x \in X(P)$ and the path leaves $x$ through $a_{k}$ or $a_{k_{1}}$ and $a_{k_{2}}$.

If $x \equiv d$ the case (a. 1) and (b. 1) also hold, but then the condition $a_{k} \notin P$ must be dropped when $a_{k} \equiv a_{l}$.

Rule (c) : If (only) two parallel arcs of $P$ enter $x$, then $x \in X(P)$ and the corresponding branch of the path ends at $x$.

Rule (d): If several arcs enter $x$ and $\omega(x)-P=\varnothing$, then $x \in X(P)$ and the corresponding branch of $P$ ends at $x$.

End: when none of the rules can be applied or when $d \in X(P)$ and all nodes, incident to the arcs of $P$, belong to $X(P)$.

2.2. A path-flow $\vec{f}^{(k)}=\left(f_{j}^{(k)}\right)$ is any flow whose support is a path $\boldsymbol{P}_{\boldsymbol{k}}$. The "support ", $\left\|\vec{f}^{(k)}\right\|$, of a vector $\vec{f}^{(k)}$ on $A$ is usually defined as the subset of arcs of $A$ for which $f_{j}^{(k)} \neq 0$.

Using the definition of flow and the rules of 2.1, it's easy to show that, if it exists, the support of a path-flow is minimal. Thus any path, support of a flow, is called elementary path.

\section{THE LABELING METHOD}

3.1. Following the method of Ford-Fulkerson, [6], the present algorithm consists of two Routines :

Routine $A$ for the labeling of nodes at the search of a flow augmenting path $P_{k}$ from $s$ to $d$;

$n^{\circ}$ mai $1974, V-2$. 

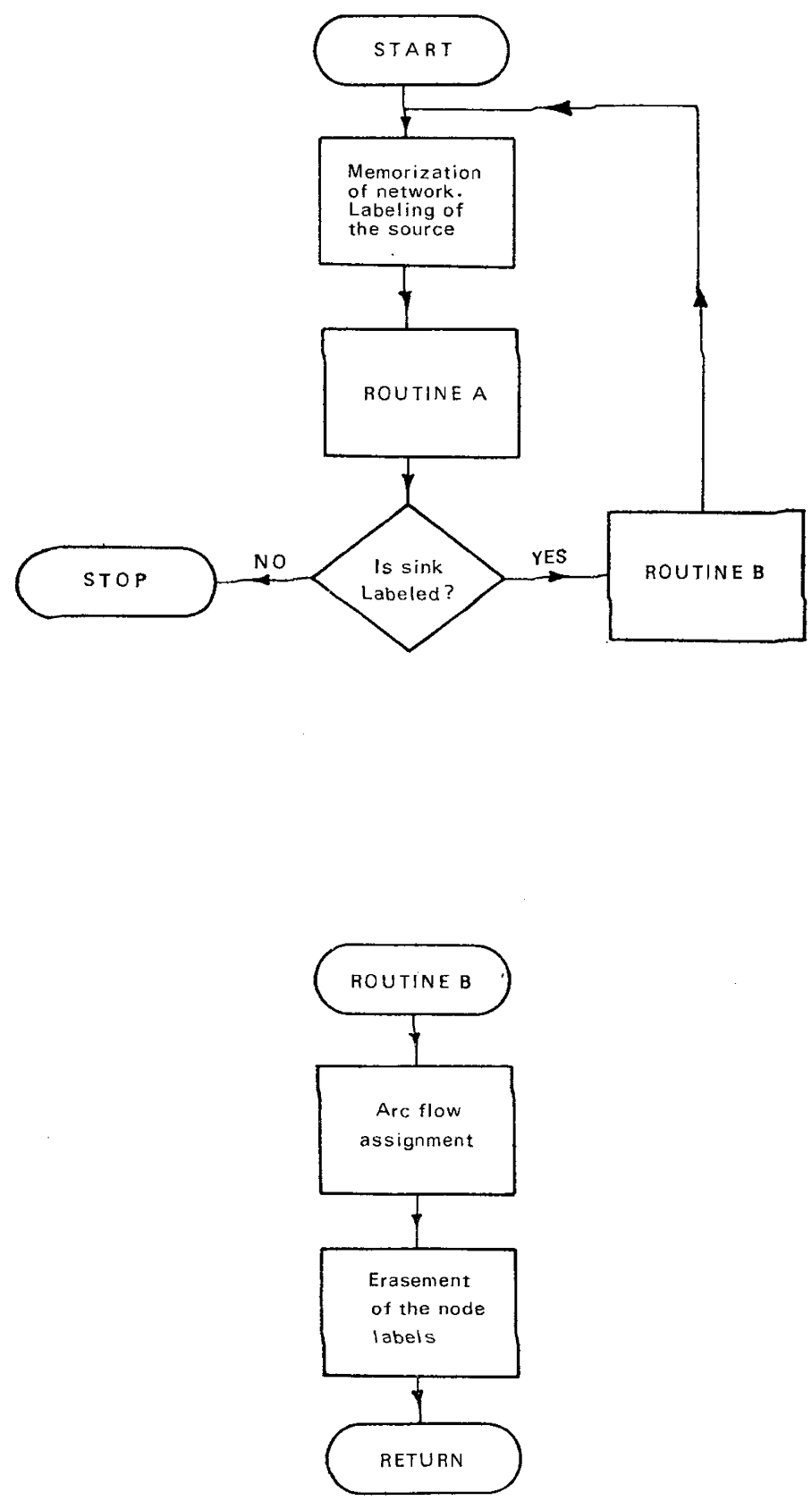

Figure $1 a$ 
Routine $B$ for increasing the flow through $P_{k}$ and evaluating the residual capacities or for concluding that the present flow is maximal.

The algorithm can start with the zero flow.

The present state of any arc $(x, y)$ is indicated by the label

$$
\left[f(x, y) / c^{s}(x, y)\right]
$$

where $f(x, y)$ is the present flow, $f \geqslant 0$, and $c^{s}$ is the conformed capacity, referred to $f(x, y)$, positive if $f(x, y) \geqslant 0$, negative if $f(x, y)<0$.

Since any incremental flow $\vec{f}^{(k)}$ can have arc flows $f_{j}^{(k)} \gtrless 0$, any arc has two residual capacities $c_{r}^{s}$, defined as

$$
c_{r}^{s}=\left\{\begin{array}{rll}
c^{s}(x, y)-f(x, y) & \text { if } & f^{(k)}(x, y) \cdot f(x, y)>0 \\
-c^{s}(x, y)-f(x, y) & \text { if } & f^{(k)}(x, y) \cdot f(x, y)<0 .
\end{array}\right.
$$

If $f(x, y)=0$, then $c_{r}^{s}(x, y) \cdot f^{(k)}(x, y)>0,\left|c_{r}^{s}\right|=c$.

\subsection{Routine A}

During this routine a node can be in one of the three states : a) unlabeled b) labeled and unscanned, c) labeled and scanned (or equilibrated).

Initially it is $\vec{f}=\overrightarrow{0}$ and all nodes are unlabeled.

The source receives the label $[/, \varepsilon(s)]$, where $\varepsilon$ is an arbitrary positive rea; number; $s$ is now in state $b$ ), and all other nodes in state a).

In general, let $x$ be any node in state b), having a label of the form $\left[y^{ \pm}, \varepsilon(x)\right]$, with $\varepsilon(x) \gtrless 0$.

We can meet different cases, corresponding to the various rules of the definition of $P_{k}$.

Case $I$ : only one arc « enter » $x$, with direction $\vec{b}$ [see rule (a)].

Here and in the following, the expression "arc entering $x$ » is for arc belonging to the support of $\vec{f}^{(k)}$, and « arc leaving $x$ » is for $\operatorname{arc} \in \omega(x)-\boldsymbol{P}_{k}$. Moreover $I$ denotes the set of indices of the choosen leaving arcs, $I=\{1\}$ for case (a. 1), $I=\{1,2\}$ for case (a. 2).

The source $s$ is always in this case ; starting from $s$, a maximal incremental flow is sent through $P_{k}$, consistent with the residual capacities $c_{r}^{s}$, following the rules given below. Such a flow cannot be augmented along $P_{k}$, but only reduced. 


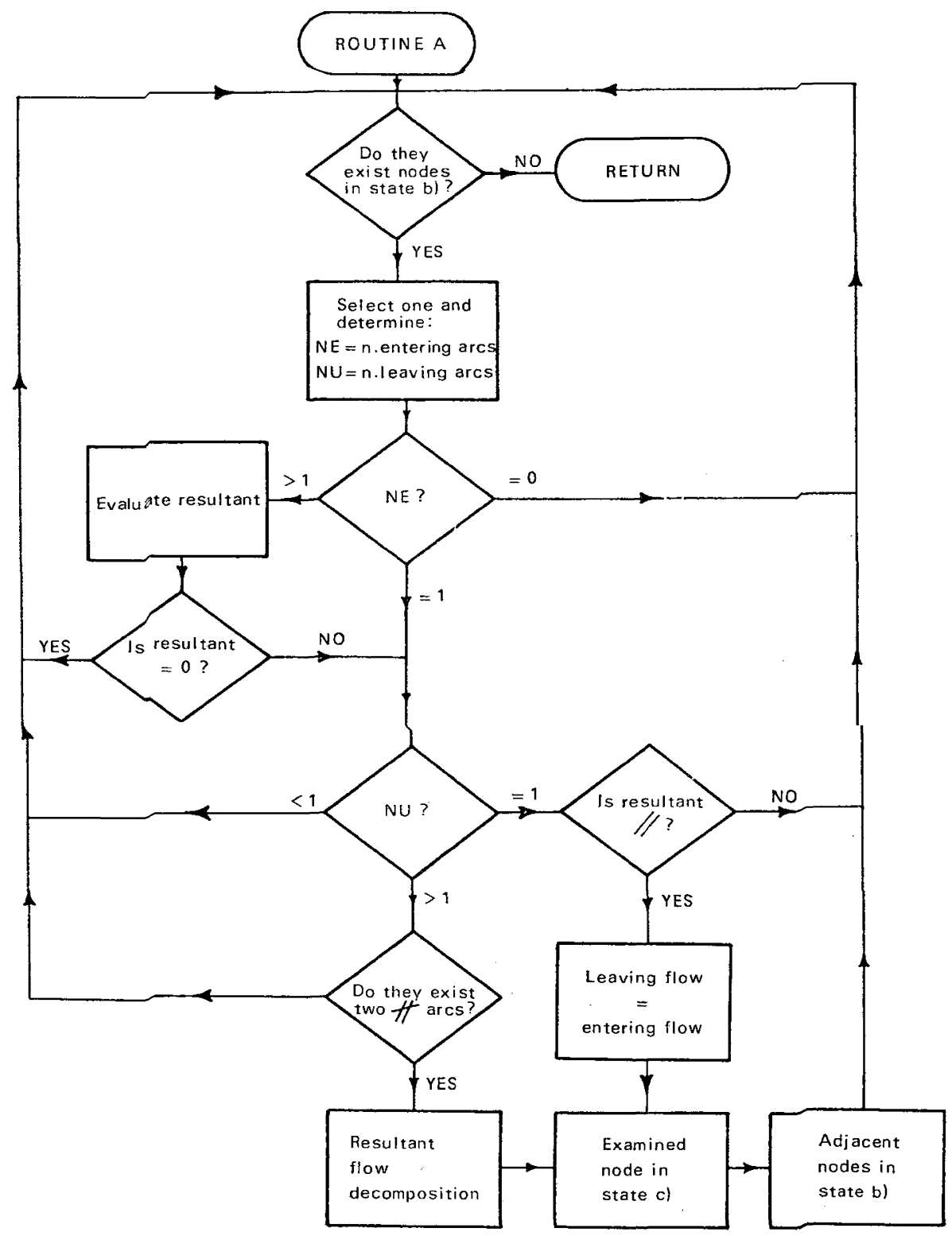

Figure $1 b$ 
If rule (a) holds, then the increment $\varepsilon(x)$ at the node $x$ is distributed along the leaving arcs in the portions $\varepsilon_{i}, i \in I$, following a conservation equation of kind

$$
\pm(\vec{b}) \varepsilon(x)+\sum_{i \in I} \pm\left(\vec{b}_{i}\right) \varepsilon_{i}=\overrightarrow{0}
$$

In both cases there exists one and only one solution with $\varepsilon_{i} \not \equiv 0$.

i) Correspondingly to the $\varepsilon_{i}$, evaluate the relative residual capacities $v_{i}$ :

$$
v_{i}=\frac{c_{r i}^{s}-\varepsilon_{i}}{\varepsilon_{i}} \quad \forall i \in I \text {. }
$$

The partial flows $\varepsilon_{i}$ are or not consistent with the capacities, according to whether it is $v_{i}>0$ or $v_{i}<0$.

If $v_{i}=0$ the arc $a_{i}$ will result saturated.

ii) If $x=s$, for any $v_{i} \forall i$,

or if $x \neq s$ and $\exists k \in I$ such that $v_{k}<0$,

then go to iv).

iii) If $x \neq s$ and $\forall i \in I v_{i}>0$,

then go to $v$ ).

iv) Select

$$
\frac{c_{r t}^{s}}{\varepsilon_{t}}=\min _{k} \frac{c_{r k}^{s}}{\varepsilon_{k}}=\lambda
$$

If $\lambda \neq 0$, reevaluate all the incremental arc-flows on $P_{k}$ with

$$
\varepsilon_{i}^{\prime}=\lambda \varepsilon_{i} \quad \forall a_{i} \in P_{k}
$$

(and denote then again $\varepsilon_{i}$ ).

Then go to v).

If $\lambda=0$, then $c_{r_{i}}^{s}=0$ and the Path is saturated.

In this case go back along $P_{k}$ searching for another breakthrough path not using the arc $a_{t}$ in the same "direction" as $P_{k}$.

v) The node $x$ is scanned.

Assign to the adjacent nodes $x_{j}$ the labels

$$
\left[x^{ \pm}, \varepsilon_{i}\left(x_{j}\right)\right]
$$

where \pm indicates an $\left\{\begin{array}{l}\text { increase of }\left|f_{i}\right| \\ \text { decrease of }\left|f_{i}\right|\end{array}\right.$

$\mathrm{n}^{\circ}$ mai 1974, V-2. 
and

$$
\varepsilon_{i}\left(x_{j}\right)= \pm \min \left[\left|\varepsilon_{i}\right|,\left|c_{r i}^{s}\right|\right]
$$

with + if $\varepsilon_{i}>0$, - if $\varepsilon_{i}<0$.

Case $I I$ : two or more arcs of $\boldsymbol{P}_{\boldsymbol{k}}$ enter $x$ and there exists one or two leaving arcs [rule (b)].

The node $x$ has been assigned several labels, $\varepsilon_{i}(x)$.

The vector-sum, $\varepsilon^{*} \vec{b}^{*}$, of the feasible entering flows must satisfy the conservation equation (7) with the leaving arcs (for the existence of $\boldsymbol{P}_{k}$ ).

If so, then $x$ is labeled and unscanned. Procede to the discussion of Case I, with $\vec{b}=\vec{b}^{*}$.

If not, then $P_{k}$ does not continue after $x$ :

(13) go back along $\boldsymbol{P}_{k}$, searching for another node $y \in N\left(P_{k}\right)$, if it exists, from where another path $P_{k}^{\prime} \neq P_{k}$ proceeds from $s$ to $d$; if it doesn't then go to vi).

Case III : two or more arcs of $P_{k}$ enter $x$ and none is leaving [rules (c) and (d)].

The equilibrium condition must be satisfied, for the existence of $P_{k}$. If not, go to (13).

vi) STOP :

Routine $A$ ends when the sink $d$ has been labeled (and scanned) and all other nodes of $P_{k}$ are in state c) [breakthrough], or when either $d$ has been labeled (and unscanned) and $\exists x \in P_{k}$ in state b), or $d$ has not been labeled (nonbreakthrough).

In the first case go to Routine $B$.

In the letter cases, GO TO 3.4.

\subsection{Routine $\mathbf{B}$}

The sink has been marked with one or more labels and the total increment is $\Sigma \varepsilon_{i}(d) \vec{b}_{i}=\vec{\varepsilon}_{l} b_{l}$.

Then go back on $P_{k}$ for a flow change : if $y$ is labeled by $\left[x^{\ddagger}, \varepsilon_{i}(y)\right] \forall i$, replace on the arc $a_{i}=(x, y)$

$$
f(x, y) \quad \text { by } \quad f^{\prime}(x, y)=f(x, y)+\varepsilon_{i}(y) .
$$

Then change the labels on arcs. Discard the old labels on nodes, and start again with ROUTINE $A$. 


\subsection{Stop}

When the non-breakthrough occurs, the present flow is maximal. It can be $\vec{f}_{\max }=\overrightarrow{0}$.

\subsection{Block diagram}

See fig. 1.

\section{6.}

The algorithm is finite since, at any new path $\boldsymbol{P}_{\boldsymbol{k}}$, at least one arc is saturated, and the arcs are at most $m+1$.

Moreover it's finite the number of combinations of $2(m+1)$ arcs, taken $l$ at $a$ time, $l=\left|P_{k}\right|$.

The proof of the algorithm is contained in the following theorem, which is an extension of a known theorem on ordinary networks, [4].

Theorem : A flow $\vec{f}$ an $G_{\vec{b}}$ is maximal if and only if it is $f_{l \max }^{*}=0$, on an associated network $G_{\vec{b}}^{*}$ obtained from $G_{\vec{b}}$ by replacing the capacities $\pm c_{i}$ by $c_{i}^{*}= \pm c_{i}-f_{i}$.

\section{COMPUTATIONAL EXPERIENCE}

For many networks, see [2], the application of the foregoing algorithm can present considerable difficulties. These are essentially due to the impossibility of stating « a priori » if a given succession of arcs constitutes or not an elementary path of $G_{\vec{b}}$. More precisely, in order to proceed to a node labeling, the program must test if any of the rules of 2.1 holds. If none of them holds, at some node $x$, we are not allowed to deny the existence of an elementary path, before all the combinations (according to the rules of 2.1), of the arcs leaving all the labeled and unscanned nodes, are tried.

The Program, in FORTRAN IV, has been tested on a computer IBM $360 / 44$. The computer times run around 60 secs for networks with $|X| \cong 10$, $|A| \cong 20$.

The Program consists of the three following parts.

\subsection{Network memorization}

The memorization of the network has been performed introducing, for every arc, a record of kind :

\begin{tabular}{|l|l|l|l|}
\hline $\mathrm{NP}(I)$ & $\mathrm{NA}(I)$ & ALFA $(I)$ & $c(I)$ \\
\hline
\end{tabular}

$n^{\circ}$ mai $1974, V-2$. 
where $\quad \mathrm{NP}(I)=$ initial node of $\operatorname{arc} I$,

NA $(I)=$ terminal node of arc $I$,

ALFA $(I)=$ inclination (degrees) of the directed arc $I$, referred to the return arc,

$$
c(I)=\text { arc capacity (positive). }
$$

The return arc is taken as directed in the usual way, i.e. from sink to source, and it's defined by a record in similar way. The network is consequently characterized, independently from the frame of reference.

\subsection{Routine A}

Whenever we get the decomposition of the entering resultant flow at a node $N$, in state b), every arc leaving $N$ take a record of kind :

\begin{tabular}{|l|l|l|l|l|}
\hline $\mathrm{NP}(J)$ & $\mathrm{NA}(J)$ & $\operatorname{ALFA}(J)$ & $c(J)$ & $\mathrm{FK}(J)$ \\
\hline
\end{tabular}

where FK $(J)=\operatorname{arc}$ flow on $a_{J}$ along $P_{k}$.

The path $\boldsymbol{P}_{k}$ is singled out from the computer, memorizing, for any arc, the order index in the succession defined by the record (15).

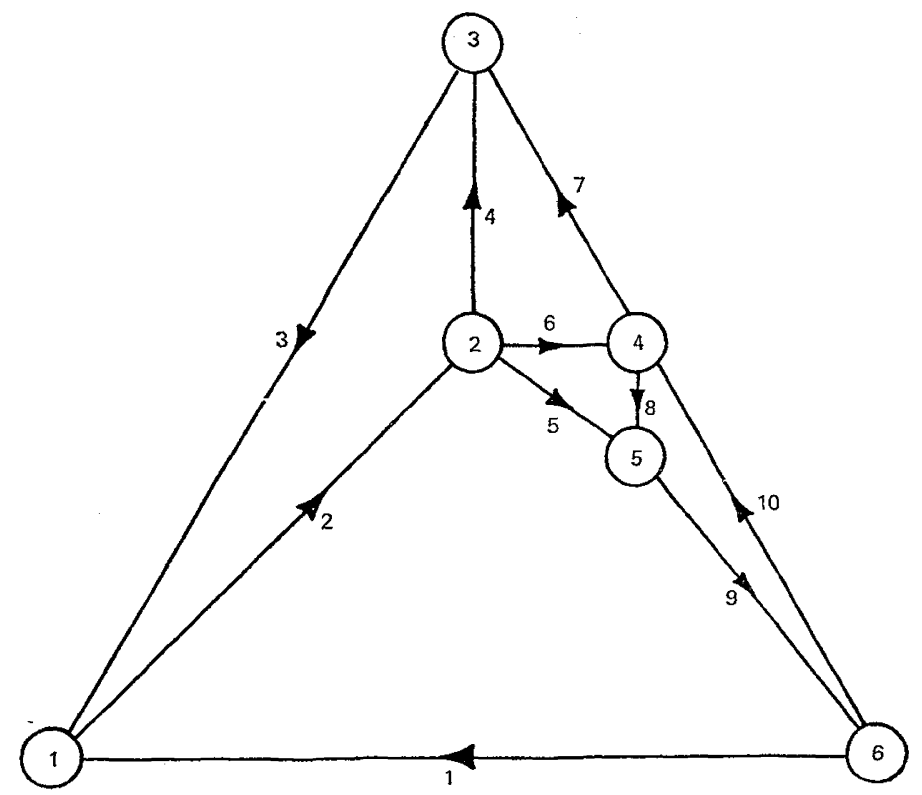

Figure 2

Revue Française d'Automatique, Informatique et Recherche Opérationnelle 
Such a device is necessary either to reduce the arc flows, conforming to (10), or to assign the arc flow in Routine B.

At the nodes, where more then two leaving arcs exist, the entering flow is first decomposed on any two arcs.

Nodes of this kind cannot be considered definitively equilibrated, i.e. in state c), but they are memorized as nodes in a « special state "; precisely : if the path $\boldsymbol{P}_{k}$ does exist from $s$ to $d$, then they enter in state c); if doesn't, the last met node must be rescanned in search of another combination of leaving arcs, for the equilibrium of the entering flow.

For some networks, with many nodes in state b), the determination of the elementary path can require rather long times of calculation. We have partially obviate such a truble, by choosing, when possible, the strategy of turning about the nodes with more then two leaving arcs. That has been realized by imposing the priority of the scanning of nodes with only two leaving arcs. Often such a device allows to avoid a combinatorial analysis.

For example, in the case of the sample problem exposed in 4.4, the Program singles out the elementary path, following the node succession $1,3,2,4,5,6$. Without the mentioned priority, at node 2 , we should have done a choice between couples of arcs $4,5,6$.

\subsection{Routine B}

Any arc, belonging to the completed path $P_{k}$, is assigned, beside the record (16), the label

$$
\text { FA }(J) \quad J=1, \ldots,\left|P_{k}\right|
$$

where FA $(J)=$ present arc flow.

Then FK $(J)$ is set equal to zero and the Program goes to 4.2.

\subsection{Sample Problem}

In the network of fig. 2 , it does exist only one elementary path; on it is optimal the flow reported in tab. 2. The features of the network are reported in tab. 1 (input of the Program). 

N. NODES -6
N. ARCS $=10$
SOURCE $=1$
SINK $=6$

\begin{tabular}{|c|c|c|c|c|c|c|c|c|}
\hline \multicolumn{2}{|c|}{ RETURN } & ARC & LEAVES & 6 & ENTERS & 1 & 0.0 (DEGREES) & \multirow[b]{2}{*}{ CAPACITY $=1.00000$} \\
\hline & N. & 2 & LEAVES & 1 & ENTEFS & 2 & 45.00 (DEGREES) & \\
\hline ARC & N. & 3 & LEAVES & 3 & ENTERS & 1 & INCL $=-120.00$ (DEGREES) & CAPACITY $=1.00000$ \\
\hline ARC & N. & 4 & LEAVES & 2 & ENTERS & 3 & 90.00 (DEGREES) & CAPACITY $=1.00000$ \\
\hline ARC & N. & 5 & LEAVES & 2 & ENTERS & 5 & INCL. $=-45.00$ (DEGREES) & CAPACITY $=1.00000$ \\
\hline ARC & N. & 6 & LEAVES & 2 & ENTERS & 4 & 0.00 (DEGREES) & CAPACITY $=1.00000$ \\
\hline ARC & N. & 7 & LEAVES & 4 & ENTERS & 3 & INCL. $=135.00$ (DEGREES) & CAPACITY $=1.00000$ \\
\hline ARC & N. & 8 & LEAVES & 4 & ENTERS & 5 & $1 \mathrm{NCL} .=-90.00$ (DEGREES) & CAPACITY $=1.00000$ \\
\hline ARC & N. & 9 & LEAVES & 5 & ENTERS & 6 & INCL. $=. .56 .65$ (DEGREES) & CAPACITY $=1.00000$ \\
\hline ARC & N. & & LEAVES & 6 & ENTERS & 4 & INCL. $=100.00$ (DEGREES) & CAPACITY $=1.00000$ \\
\hline
\end{tabular}

\section{TABLE 1}

RESULTS

FLOW PATH N. 1 COMPLETE

$\begin{array}{lll}\text { ARC N. } 1 & \text { MAX. FLOW }=0.2679487 \text { E } 00 \\ \text { ARC N. } 2 & \text { MAX. FLOW }=0.8965754 \text { E } 00 \\ \text { ARC N. } 3 & \text { MAX. FLOW }=-0.7320511 \text { E } 00 \\ \text { ARC N. } 4 & \text { MAX. FLOW }=0.1000000 \text { E } 01 \\ \text { ARC N. } 7 & \text { MAX. FLOW }=-0.5176360 \text { E } 00 \\ \text { ARC N. } 5 & \text { MAX. FLOW }=0.5176407 \text { E } 00 \\ \text { ARC N. } 6 & \text { MAX. FLOW }=0.2679466 \text { E } 00 \\ \text { ARC N. } 8 & \text { MAX. FLOW }=0.1901358 \text { E } 00 \\ \text { ARC N. } 9 & \text { MAX. FLOW }=0.6658028 \mathrm{E} 00 \\ \text { ARC N. } 10 & \text { MAX. FLOW }=0.5647412 \mathrm{E} 00\end{array}$

TABLE 2 


\section{REFERENCES}

[1] Berge C. and Ghoulla-Houri A., Programmes, jeux et réseaux de transport, Dunod, Paris, 1962.

[2] BorReANI A., « Two-dimensional networks » ORC 70-6, february 1970. Operations Research Center, University of California Berkeley.

[3] Charnes A. and COOPER W. W., Management models and industrial applications of linear programming, vol. II, J. Wiley \& Sons, 1961.

[4] Dantzig G. B., Linear programming and extensions. Princeton University Press, 1963.

[5] Dorn W. S. and Greenberg H. J., "Linear Programming and Plastic Limit Analysis of Structures ». Quarterly of Applied Math., vol. 15, pp. 155-167, 1957.

[6] Ford L. R. and Fulkerson D. R., Flows in networks. Princeton University Press, 1962.

[7] Fulkerson D. R., « Networks, Frames, Blocking Systems ». Mem. RM - 5368-PR, may 1967. Rand Corporation.

[8] Ostanello-BorreanI A., «Flussi sui reticoli : alcuni recenti sviluppi ». Atti Giornate AIRO-Torino, 1971.

[9] Prager W., "Mathematical Programming and theory of Structures ». J. of the Society for Industrial and Applied Mathematics, vol. 13, $\mathrm{n}^{\circ} 1$, pp. 312-332, 1965. 